\title{
Avaliação do Ambiente Virtual de Aprendizagem Moodle sob diferentes visões: aspectos a considerar
}

Patricia Mariotto Mozzaquatro - PPGI/ UFSM - patriciamozzaquatro@gmail.com Roseclea Duarte Medina - PPGI/ UFSM - roseclea.medina@gmail.com

\section{Resumo}

Esse artigo tem como principal objetivo apresentar diferentes visões do Ambiente Virtual de Aprendizagem Moodle utilizado pelas Instituições de Ensino Superior: UFSM e UFPel, definindo funcionalidades oferecidas e comparando-as, como também, destaca a importância de avaliar AVAs, mediante o crescimento explosivo nos últimos anos dos Cursos na modalidade de educação a distância. Utilizou-se a Análise de Questionários e Observação, com o objetivo de proporcionar a utilização de um mesmo ambiente por universidades distintas, a fim de ampliar as experiências com AVAs e desenvolver condições para realizar avaliações críticas. Os resultados também foram submetidos aos gestores de EAD e a equipe de TI para tomarem conhecimento das sugestões de melhorias nos ambientes utilizados nas Instituições (UFSM e UFPel).

Palavras - Chaves: educação à distância, moodle, ambientes virtuais de aprendizagem, avaliação.

\section{Evaluation of Virtual Learning Environment Moodle under different visions: aspects to consider}

\begin{abstract}
This article has the main objective of presenting different visions of the Virtual Learning Environment Moodle used by Higher Education Institutions: UFSM and UFPel, defining features offered and comparing them, but also highlights the importance of assessing AVAS by growth explosive in recent years of courses in the distance mode of education. Using the analysis of questionnaires and observation, aiming to provide for the use of the same environment for different universities in order to extend the experiment with AVAs and developing conditions for carrying out criticism. The results were also submitted to the managers of EAD and the IT team to take note of the suggestions for improvements in environments used in institutions (UFSM and UFPel).
\end{abstract}

Keywords: distance education, moodle, virtual environments for learning, evaluation.

\section{Introdução}

Nas últimas décadas, a educação a distância (EAD) tomou um novo impulso que favoreceu a disseminação e a democratização do acesso à educação em diferentes níveis e formas de interação e aprendizagens. Os processos estão, cada vez mais, se articulando através dos ambientes virtuais de aprendizagem (AVAs). No contexto acadêmico, esta realidade cria novas oportunidades para os educadores compartilharem com os alunos o acesso à informações. Nesse sentido, o advento das tecnologias de informação e comunicação (TICs) trouxe novas perspectivas para a EAD, levando as Instituições de Ensino, empresariais e os profissionais de instruction design a se dedicarem ao desenvolvimento de cursos a distância com suporte em AVAs. Conforme Primo (2008), 
Apesar das novas tecnologias oferecerem cada vez mais recursos para a aprendizagem, a desorganização no planejamento na apresentação de um material multimídia, e a utilização inadequada de ferramentas disponíveis num AVA, podem causar desorientação do usuário e/ou até mesmo desmotivá-lo a se engajar na atividade proposta.

Quando se decide pela utilização de um AVA é preciso estabelecer critérios coerentes que sejam mais adequados ao processo educacional. Como analisar pontos positivos e negativos? O que deve ser relevante? Que parâmetros devem nortear esta escolha?

É nesse contexto de variáveis que se procura melhor avaliar um ambiente, podendo assim, possibilitar e promover situações de aprendizagem que mobilizem os estudantes a gerar significados e, por conseguinte, melhorar a construção de conhecimentos de forma autônoma.

A necessidade de aprofundamento no tema avaliação de AVAs na EAD, definiu os objetivos deste artigo, que foi avaliar, comparar e analisar as customizações realizadas em dois ambientes de estudo - Modular Object-Oriented Dynamic Learning Environment (Moodle), utilizado pelas Instituições de Ensino Superior Universidade Federal de Santa Maria (UFSM) e Universidade Federal de Pelotas (UFPel), a partir da análise de questionários e da observação do comportamento dos alunos com relação a utilização do ambiente, aspectos como funcionalidades, ergonomia, usabilidade e ferramentas integrantes dos AVAs Moodle, favorecendo com isso, melhorias consideráveis nas habilidades e competências necessárias para a realização de avaliações críticas/técnicas de AVAs.

\section{Ambientes Virtuais de Aprendizagem}

No atual cenário é fundamental que a Educação integre-se à sociedade do conhecimento. Uma das formas de realizar essa tarefa é desenvolver estratégias de aprendizagem, mediadas por tecnologia da informação (TI), um exemplo são os AVAs. Estes ambientes encontram-se em grande expansão, nas mais diversas instituições: acadêmicas, empresariais e tecnológicas, com objetivo de ser uma ferramenta de $E$ learning, possibilitando a capacitação de profissionais e estudantes.

A expressão Ambiente Virtual de Aprendizagem, de acordo com Almeida (2004), "relaciona-se à sistemas computacionais, destinados ao suporte de atividades mediadas pelas tecnologias de informação e comunicação". Permitem integrar múltiplas mídias e recursos, apresentam informações de maneira organizada, proporcionam interações entre pessoas e objetos de conhecimento, visando atingir determinados objetivos.

Os AVAs podem ser empregados como suporte para sistemas de EAD, bem como servir de apoio às atividades presenciais de sala de aula e/ou diferentes ambientes por meio da internet ou intranet.

No contexto desta pesquisa foi considerado o AVA Moodle, apresentado na seção a seguir.

\subsection{Ambiente Virtual de Aprendizagem Moodle}

Conforme os autores Ribeiro e Mendonça (2007),

O AVA Modular Object Oriented Distance Learning (Moodle) é uma plataforma, Open Source, ou seja, pode ser instalado, utilizado, modificado e mesmo distribuído. Seu desenvolvimento objetiva o gerenciamento de aprendizado e de trabalho colaborativo em ambiente virtual, permitindo a 
criação e administração de cursos on-line, grupos de trabalho e comunidades de aprendizagem.

Está disponível em mais de setenta idiomas e possui uma variedade de módulos com diferentes níveis de estabilidade. Apresenta uma interface adaptada a browsers.

Este AVA segundo Garcia e Lacleta, "apresenta estrutura modular, ampla comunidade de desenvolvedores, grande quantidade de documentação, disponibilidade, escalabilidade, facilidade de uso, interoperabilidade, estabilidade e segurança". Por ser um ambiente que oferece diversas ferramentas e alto grau de customização vem sendo utilizado por várias instituições de Ensino.

\section{Avaliação de Ambientes Virtuais de Aprendizagem}

A avaliação de AVAs pode tomar como base para sua investigação, as condições em que a aprendizagem se realiza (estrutura), os modos pelos quais os estudantes são capazes de interagir sendo apoiados nas suas atividades (processos) e o alcance dos objetivos e das metas propostas (resultados).

Os instrumentos de avaliação em AVAs são componentes fundamentais e permitem dar feedback ao desenvolvedor e ao formador sobre os aspectos de usabilidade, ergonomia, confiabilidade, acessibilidade e interação e aspectos pedagógicos. A interface destes sistemas deve ser amigável e intuitiva, bem como, facilitar o seu uso e diminuir o processo exaustivo da busca de acesso a informação pelo usuário. Avaliar AVAs é uma tarefa complexa, pois além de estarem em constantes estudos e evolução, contemplam variáveis de tecnologia e de aprendizagem.

Para a autora Oliveira (2001),

A acessibilidade, o intuito e a facilidade de uso em um sistema podem ser considerados como os fatores determinantes para a utilização ou não de um serviço de informação, requerendo constante feedback para que esses serviços possam ser planejados e atendam as necessidades presentes dos seus usuários.

\subsection{Métodos de avaliação de Ambientes Virtuais de Aprendizagem}

Benigno e Trentin (2000), afirmam que:

Na avaliação de AVAs, é necessário dispor de dados sobre características individuais dos participantes (observação do comportamento), do ambiente de aprendizagem, participação, comunicação, materiais e da tecnologia utilizada.

Foram utilizados os seguintes métodos de avaliação: observação e questionários, apresentados a seguir.

Para o autor Dixon (2001),

A aplicação de questionários pode ser realizada de forma presencial ou online, apresentando as seguintes vantagens: rapidez na coleta dos dados, uso de grandes amostras, menor custo de administração e processamento e taxas de retorno mais altas.

A observação dos usuários pode ser de duas formas, direta ou indireta. Na forma direta o avaliador observa o comportamento do usuário, como seqüência de ações. $\mathrm{O}$ usuário pode modificar o comportamento pelo fato de estarem sendo observado. $\mathrm{Na}$ observação indireta são coletadas informações de forma automática, não interferindo no comportamento do usuário.

\subsection{Critérios ergonômicos para avaliação de interfaces de AVAs}


De acordo com o autor Wisner (1995),

A ergonomia é o conjunto dos conhecimentos científicos necessários à concepção de ferramentas, máquinas e dispositivos que possam ser utilizados com conforto, segurança e eficácia. A abordagem ergonômica baseia - se no princípio básico de que o trabalho deve se adaptar ao homem. A transferência deste princípio para a informática gerou um enunciado mais específico: adaptar o computador ao usuário, e não o contrário.

Os Critérios Ergonômicos constituem um conjunto de qualidades ergonômicas que as interfaces humano-computador (IHC) deveriam apresentar, foram desenvolvidos por dois pesquisadores de língua francesa, Dominique Scapin e Christian Bastien, ligados ao Institut National de Recherche en Informatique et en Automatique da França (INRIA) em 1993. Segundo Bastien e Scapin (2003),

são apresentados os seguintes critérios ergonômicos: Condução, Carga de trabalho, Controle Explícito, Adaptabilidade, Gestão de Erros, Homogeneidade /Coerência/ Consistência, Significado dos códigos e denominações e Compatibilidade.

\section{Metodologia}

Para este trabalho de pesquisa foram selecionados sessenta usuários, acadêmicos do Curso de Licenciatura em Matemática da UFPel (vinte e sete), acadêmicos dos Cursos de Letras Português - Literaturas (catorze) e Curso de Pedagogia da UFSM (dezenove), de duas Instituições de Ensino Superior pertencentes ao Sistema UAB, que ministram curso de EaD no Pólo Educacional Superior de Restinga Sêca.

O experimento consistiu na criação e aplicação do instrumento de avaliação e na observação dos alunos durante a interação com o AVA Moodle nas duas Instituições. O instrumento desenvolvido consistiu de questões objetivas sobre o ambiente em que as respostas poderiam ser "muito bom", "bom", "regular", "ruim" e "péssimo", bem como questões abertas de livre resposta.

Foi analisada a página do Laboratório de Usabilidade (LabIUtil), para escolha das possíveis questões referentes à usabilidade e elaborado o questionário avaliativo a partir da análise de três modelos de avaliação: Modelo de Eliane Schlemmer "et al". (2007), Checklist apresentado por Gildásio Guedes (2005) e Andréa Filatro (2004), de acordo com os seguintes critérios: interface com o usuário (apresentação geral, estrutura, desempenho), funcionalidade do ambiente, usabilidade, mecanismos de comunicação, mecanismos de cooperação, ferramentas de interação (síncrona e assíncrona), aspecto indutivo no uso e layout da tela.

O trabalho consistiu na avaliação da Plataforma Moodle utilizada pelas duas Instituições, onde foi aplicado o instrumento de pesquisa desenvolvido.

O processo foi desenvolvido nas seguintes etapas: interação dos acadêmicos com os dois Ambientes Virtuais de Aprendizagem; observação dos usuários nos ambientes citados e discussões; elaboração do questionário de avaliação; aplicação do questionário avaliativo; análise quantitativa dos resultados; apresentação dos resultados obtidos e elaboração das conclusões.

\section{Resultados e Discussão}

Foram avaliadas as características funcionais e não funcionais dos ambientes avaliados. Observou-se que as formas de avaliação utilizadas (observação e questionário) mostraram-se eficazes para o objetivo proposto. 
Os resultados (em números) decorreram da compilação do formulário aplicado conforme demonstrados a seguir:

Primeiramente é necessário observar que os alunos da pesquisa encontram-se na faixa etária de dezoito a vinte e cinco anos, e mais de $75 \%$ são do sexo feminino.

A Figura 1 apresenta formas de interação no ambiente. Os alunos das duas Instituições de Ensino consideram a forma de interação no ambiente boa.

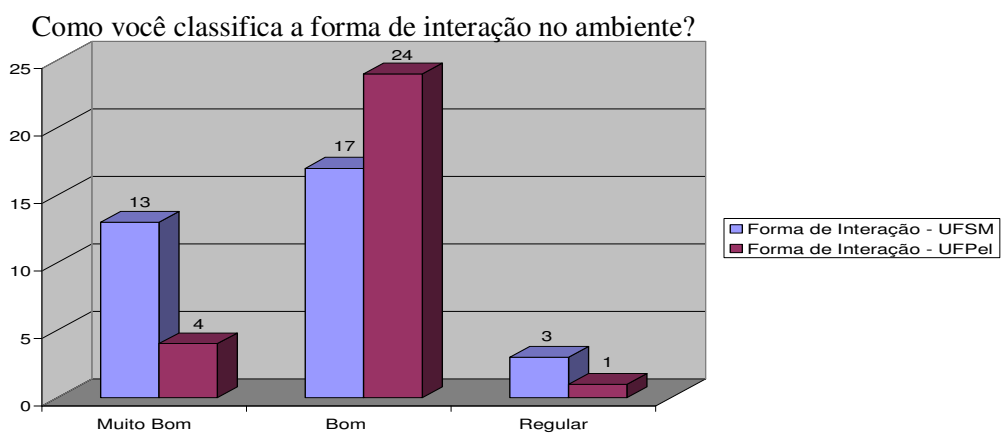

Figura 1 - Formas de interação

Outros aspectos destacados foram em relação aos componentes da plataforma Moodle que mais contribuíram para a aprendizagem. Constatou-se que a ferramenta de interação assíncrona mais usada foi fórum, seguida de livros (alunos da UFSM) e Questionários (alunos da UFPel), expostos na Figura 2.

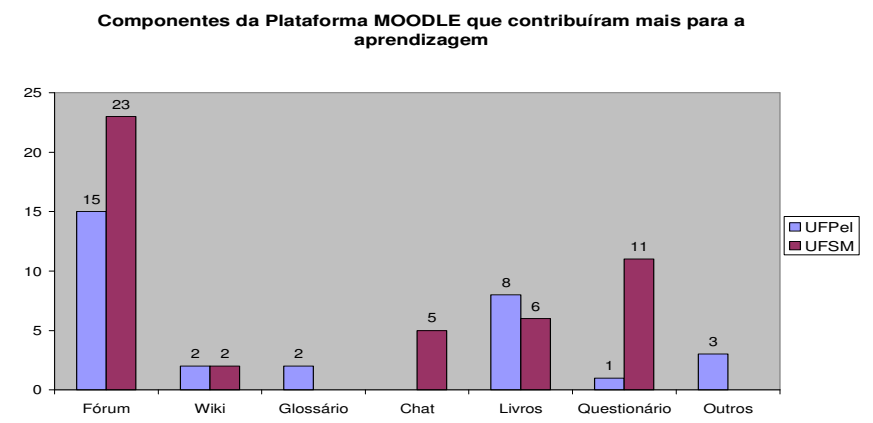

Figura 2 - Componentes que mais contribuíram para a aprendizagem

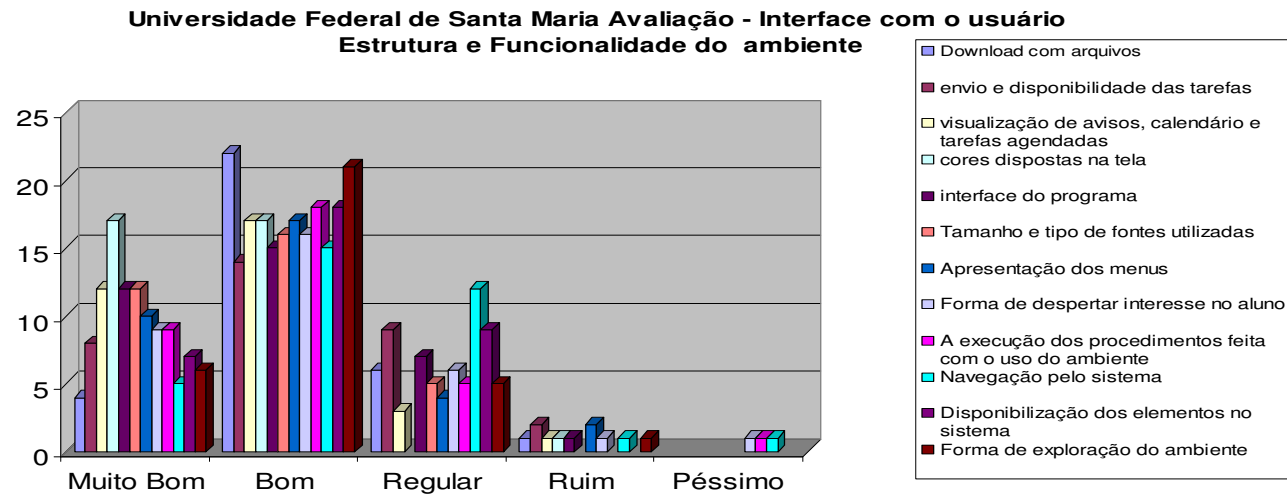

Figura 3 - Avaliação da interface com o usuário, estrutura e funcionalidade do ambiente 
Universidade Federal de Pelotas Avaliação - Interface com o usuário Estrutura e Funcionalidade do ambiente

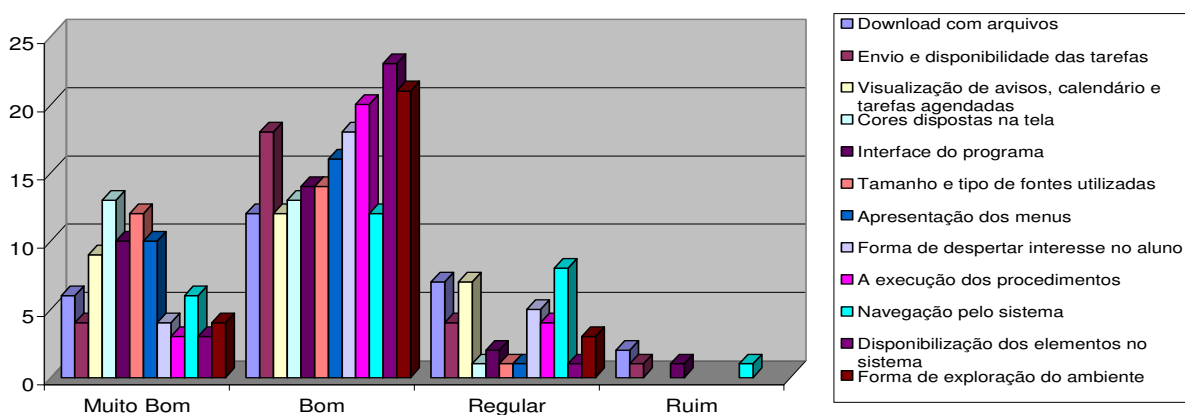

Figura 4 - Avaliação da interface com o usuário, estrutura e funcionalidade do ambiente

Em relação a interface com o usuário, estrutura e funcionalidades do ambiente, apresentadas nas Figuras 3 e 4, constatou-se que, quanto às cores apresentadas e sua disposição na tela pelos dois ambientes encontram-se muito boas, a apresentação inicial do ambiente, ou seja, a interface do ambiente é boa. Quanto ao tipo de fonte utilizada, também foi considerada boa. $\mathrm{O}$ download de arquivos, apresentação de menus de forma clara, navegação pelo sistema e envio de tarefas enquadram-se na categoria bom pelas duas Instituições de Ensino. Os alunos da UFSM sugeriram, como melhoria, o aumento no upload para envio de arquivos.

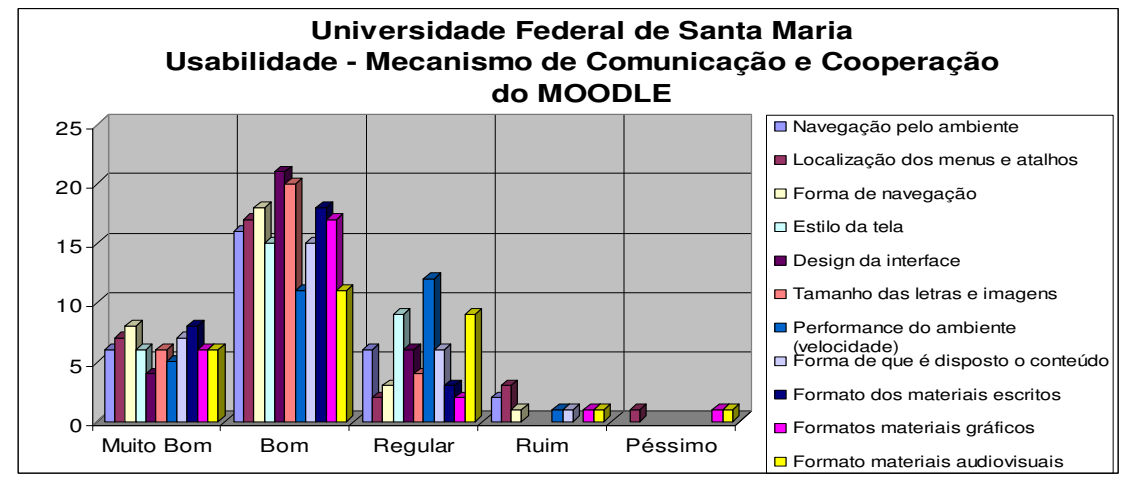

Figura 5 - Usabilidade, mecanismo de comunicação e cooperação do Moodle- UFPel

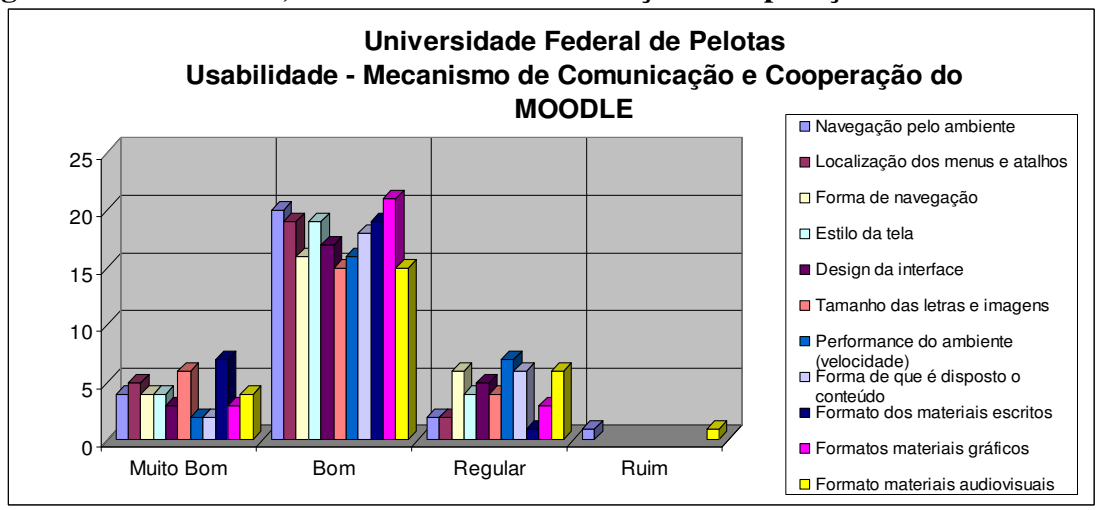

Figura 6 - Usabilidade, mecanismo de comunicação e cooperação do Moodle- UFSM

As Figuras 5 e 6 nos mostram que nos aspectos relacionados a navegação, a maioria considera boa, pois as telas proporcionam uma navegação de forma rápida e agradável. O design da interface forma um todo, todavia, foi considerado bom. $\mathrm{O}$ aspecto relacionado ao desempenho do ambiente (velocidade) a maioria dos entrevistados da UFPel considera bom, já os da UFSM consideram regular. Em relação 
ao formato dos materiais, tanto audiovisuais, quanto os gráficos ou desenhos, foram considerados bons, pelos alunos das duas Instituições de Ensino.

Para melhor embasar a pesquisa foram feitos questionamentos aos acadêmicos. Ratificando ao que colocamos anteriormente, a ferramenta fórum foi considerada a que mais contribui para o processo de aprendizagem. Segundo as suas opiniões: "Sim, pois cada um tem uma forma de pensar, socializar diferente dos outros, expor sua opinião, abre caminhos para novas idéias". (Aluno UFPel); "Sim, pois com o uso do fórum percebemos as várias formas e ponto de vista sobre um mesmo conteúdo." (Aluno UFSM). Outro aspecto abordado foi sobre a avaliação do curso a distancia através do ambiente Moodle em relação à aprendizagem. Os alunos expõem o seguinte: $\mathrm{O}$ ambiente Moodle é uma ferramenta que pode ou não contribuir para a aprendizagem, depende do conhecimento que o indivíduo possui sobre seu funcionamento." (Aluno UFPel); "Muito bom, pois com o curso a distância temos que ter autonomia e ir em busca do conhecimento." (Aluno UFSM).

Embora reconhecendo que o AVA Moodle, é um importante e valioso instrumento para a EAD permitindo uma postura cooperativa de interação, constatou-se que nas duas Instituições existem ainda, possibilidades de melhorias. No que se refere a formação integral do aluno podemos evidenciar a necessidade de ser incluído no ambiente virtual da UFSM o recurso geração de relatórios, o qual possibilita ao aluno o acompanhamento da realização das atividades (fórum, Chats, Trabalhos, Leitura de arquivos), bem como seu rendimento (notas em provas e trabalhos). Outro aspecto citado como melhoria, refere-se a um aumento no upload para envio de arquivos, principalmente com extensões do tipo ppt, pdf, html, dentre outras.

\section{Considerações Finais}

O instrumento de pesquisa que serviu como ferramenta de avaliação mostrou-se válido e viável, pois através do mesmo, foi realizada a análise e avaliação da estrutura e organização do AVA Moodle, utilizado em duas Instituições de Ensino Superior. Foi possível compreender as necessidades dos alunos envolvidos, vislumbrando possíveis melhorias amparadas nas opiniões colocadas.

As formas de interação no ambiente foram consideradas boas e a ferramenta de interação que mais contribuiu foi o fórum, seguido da ferramenta de livros (alunos da UFSM) e Questionários (alunos da UFPel).

Em relação a interface e funcionalidades do ambiente, constatou-se que, quanto a apresentação inicial do ambiente, ou seja, a interface do ambiente é boa. O envio e disponibilidade das tarefas, forma de despertar interesse no aluno, disponibilidade dos elementos e forma de exploração no ambiente, enquadraram-se na categoria bom pelas duas Instituições de Ensino. Quanto ao download de arquivos, também foi considerado bom nos dois ambientes.

Os aspectos relacionados a navegação, a maioria considerou boa, pois as telas proporcionaram uma navegação de forma rápida e agradável. O design da interface forma um todo, todavia, foi considerado bom. $\mathrm{O}$ aspecto relacionado ao desempenho do ambiente (velocidade) a maioria dos entrevistados da UFPel considerou bom, já os da UFSM consideraram regular - isso influenciou na substituição do hardware do servidor por um mais robusto. Em relação ao formato dos materiais, tanto audiovisuais, quanto os gráficos ou desenhos, foram considerados bons, pelos alunos das duas Instituições de Ensino. 
Embora reconhecendo que o AVA Moodle, é um importante e valioso instrumento para a educação a distância permitindo uma postura cooperativa de interação, constatou-se que nas duas Instituições existem ainda, possibilidades de melhorias. No que se refere a formação integral do aluno podemos evidenciar a necessidade de ser incluído no AVA da UFSM o recurso de geração de relatórios, o qual possibilita ao aluno o acompanhamento da realização das atividades (fórum, Chats, Trabalhos, Leitura de arquivos), bem como seu rendimento (notas em provas e trabalhos). Outro aspecto citado como melhoria, refere-se a um aumento no upload para envio de arquivos, principalmente com extensões do tipo ppt, pdf, html, dentre outras.

Este trabalho almeja contribuir com novos olhares sobre AVAs, seja como elemento auxiliar no complexo processo de escolha de um ambiente, bem como, no suporte para as necessidades de customização dos ambientes, resultando em AVAs mais apropriados as necessidades dos alunos. Diante disso, não se tem a pretensão de apresentar resultados conclusivos sobre o AVA Moodle avaliado nas duas Instituições, mas servir para orientar os trabalhos iniciais no contexto de avaliação de AVAs. Como continuidade do trabalho, a partir dos resultados alcançados, será realizada uma nova rodada de avaliações considerando alterações que se mostraram necessárias, percebidas durante o trabalho, como por exemplo, expansão do instrumento de avaliação e disponibilização de mais tempo para interação e utilização dos AVAs em processo de avaliação principalmente com o perfil de Administrador.

\section{Referências}

ALMEIDA, Maria Elizabeth Bianconcini de. (2004). Tecnologia e educação a distância: abordagens e contribuições dos ambientes digitais e interativos de aprendizagem. Disponível em:< http://www.anped.org.br/reunioes/26/trabalhos /ma riaeliza bethalmeida.rtf>. Acesso em: 10 set. 2008.

BASTIEN, Christian; SCAPIN, Dominique. (2003). Critérios Ergonômicos para Avaliação de Interfaces Homem-Computador. Disponível em: < http://www.labiutil.inf.ufsc.br/CriteriosErgonomicos/LabIUtil2003Crit/100conduc.html >. Acesso em: 20 set. 2008.

BENIGNO, V.; TRENTIN, G. The evaluation of online courses. Journal of Computer Assisted Learning, v. 16, p. 56-70, 2000.

BOAS, Vitor Villas. Ambientes Virtuais de Aprendizagem - Moodle x Plataformas Pagas. Disponível em: <http://imasters.uol.com.br/artigo/4600/elearning /ambientes_virtuais_de_aprendizagem_-_moodle_x_plataformas_pagas/>. Acesso em 26 ago. 2008.

CORREIA, Secundino. Comparação de Ambientes Virtuais de Aprendizagem estratégias de avaliação. Disponível em: <http://conteudo.thegraal.net/DL CW06_papers/DLCWPDF/ secundinoartigo_v2.pdf>. Acesso em 20 ago. 2008.

Computer Assisted Learning, v.16, p. 59-70, 2000.

DIXON, J. Evaluation tools for flexible delivery (workshop version). Melbourne: TAFE frontiers, 2001.

FERNANDES, Gildásio Guedes. Avaliação da usabilidade da Interface Humano Computador do Ambiente Colaborativo de Aprendizagem a Distância (Eproinfo). 
Disponível em: <http://www.ufpi.br/mesteduc/eventos/ivencontro/GT17 avaliacao_usabilidade.pdf $>$. Acesso em 10 ago. 2008.

FILATRO, Andréa. Design instrucional contextualizado: educação e tecnologia. São Paulo: Editora SENAC, 2004

GARCIA, Pablo López; LACLETA, María Luisa Sein-Echaluce. A revolução pedagógica: o meio Moodle. Disponível em: <http://contenidos.universia .es/html_trad/traducirEspecial/params/especial/bc/seccion/6/titulo/REVOLUCIONPEDAGOGICA-ENTORNO-MOODLE.html>. Acesso em: 25 ago. 2008.

GONZALES, Mathias. Fundamentos da Tutoria em Educação a Distância. Avercamp, São Paulo, 2005.

GUEDES, Gildásio. Um checklist para avaliar uma plataforma virtual de aprendizagem. In: ALBUQUERQUE, L. B. (Org). Currículos Contemporâneos: formação, diversidade e identidade em transição. Fortaleza: Editora UFC, 2005.

Laboratório de Usabilidade: Critérios Ergonômicos. Disponível em:< http://www.labiutil.inf.ufsc.br/>. Acesso em 03 set. 2008.

MATIAS, Márcio. (1995). Avaliação ergonômica de interfaces. Disponível em:<http://www.eps.ufsc.br/disserta/matias/capit_3/cap3_mat.htm>. Acesso em: 15 out. 2008.

MATIAS, Márcio. Checklist: Uma ferramenta de suporte à avaliação ergonômica de interfaces. Florianópolis, 1995 (dissertação de mestrado).

MOODLE. Modular Object-Oriented Dynamic Learning Environment. Disponível em:< http://moodle.org >. Acesso em 10 jul. 2008.

MOODLE. “Modular Object-Oriented Dynamic Learning Environment. Disponível em: < http://cead.ufsm.br/MOODLE/login/index.php>. Acesso em:18 jul. 2008.

MOODLE. “Modular Object-Oriented Dynamic Learning Environment. Disponível em: <http://200.132.97.7/login/index.php>. Acesso em:18 jul. 2008.

OLIVEIRA, Elaine Rosangela de. (2001). Avaliação Ergonômica de Interfaces da Scielo - Scientific Electronic Library Online. Disponível em: < http://teses.eps.ufsc.br/defesa/pdf/4705.pdf>. Acesso em: 10 set. 2008.

Pólo educacional Superior de Restinga Seca. (2007). Disponível em: $<$ http://www.uabrestingaseca.com.br >. Acesso em: 10 mai. 2008.

PRIMO, Lane. (2008). Auto-Avaliação na Educação a Distância uma alternativa viável. Disponível em: < http://www.prodepa.gov.br/sbc2008/anais/pdf/arq0132.pdf>. Acesso em: 15 ago. 2008.

RIBEIRO, Elvia Nunes; MENDONÇA, Gilda Aquino de Araújo e MENDONÇA, Alzino Furtado. (2007). A importância dos Ambientes Virtuais de Aprendizagem na busca de novos domínios na EAD. Disponível em: < http://www.abed.org.br/congresso2007/tc/4162007104526AM.pdf>. Acesso em: 18 jul. 2008.

ROCHA, Heloísa; BARANAUSKAS, Maria. Design e avaliação de interfaces humano-computador. São Paulo, IME-USP, 2000 (Escola de Computação, 2000), $242 \mathrm{p}$. 
SCHLEMMER, Eliane; SACCOL, Amarolinda; GARRIDO, Susane. (2007). Um Modelo Sistêmico de Avaliação de Softwares para Educação a Distância como apoio à Gestão de EAD. Disponível em: < http://www.ead.fea.usp.br/cadpesq/arquivos/493.pdf.>. Acesso em: 08 out. 2008.

Universidade Aberta do Brasil. Disponível em: < http://uab.capes.gov.br/index.php > Acesso em:15 abr. 2008.

WISNER, Alain. (1995). As transformações do trabalho e os desafios teóricosmetodológicos da ergonomia: Desafios teóricos metodológicos da ergonomia. Disponível em: <http://www.unb .br/ip/labergo/sitenovo /Julia/Artigos/paraosite /DTM E.PDF>. Acesso em: 25 set. 2008. 